\title{
A LEMMA FOR NEGATIONLESS PROPOSITIONAL LOGICS AND ITS APPLICATIONS
}

\section{TOSIYUKI TUGUÉ}

(To Professor Kiyoshi Noshiro on the occasion of his 60th birthday.)

In this note, we treat the positive sentential logic LPS and the primitive sentential logic (the positive implicational calculus of Hilbert) LOS ${ }^{1}$. LOS has 'implication' as the only logical symbol and is a subsystem of LPS. LPS can be formulated as follows:

Proposition letters: $p, q, r, \ldots$; or $p_{1}, p_{2}, p_{3}, \ldots$.

Logical symbols: $\rightarrow, \vee$ and $\Lambda$.

Formation rule: as usual.

Axiom schemata:

A 1.

$$
A \rightarrow(B \rightarrow A),
$$

A 2.

$$
(A \rightarrow B) \rightarrow((A \rightarrow(B \rightarrow C)) \rightarrow(A \rightarrow C)),
$$

A 3.

$$
A \rightarrow A \vee B, \quad B \rightarrow A \vee B,
$$

A 4 .

$$
(A \rightarrow C) \rightarrow((B \rightarrow C) \rightarrow(A \vee B \rightarrow C)),
$$

A 5.

$$
A \rightarrow(B \rightarrow A \wedge B),
$$

A 6.

$$
A \wedge B \rightarrow A, \quad A \wedge B \rightarrow B
$$

Inference rule: Modus Ponens.

To the system LPS or LOS, by adding Peirce's law:

A 7.

$$
((A \rightarrow B) \rightarrow A) \rightarrow A,
$$

we obtain the corresponding 'classical' logic, which is denoted by LQS1) or LOQS, respectively.

Received September 6, 1966.

1) The reference notation LOS to the primitive sentential logic is found in Ono [7] where it is called the sentence-logical part of the primitive logic. Church [1] refers to LOS and LPS by $P^{+}$and $P^{P}$, respectively. As for the primitive logic LO, the intuitionistic positive logic LP and the classical positive logic LQ, see Ono [6]. cf. also Curry [2] and Lorenzen [3] as to the positive logics. 
Now, we take the ordinary valuation $v(A)$ for the formulae on the values $\{0,1\}$ :

$$
\begin{aligned}
& v(A \rightarrow B)=\left\{\begin{array}{l}
0 \text { if } v(A) \geqq v(B), \\
1 \quad \text { otherwise, }
\end{array}\right. \\
& v(A \vee B)=\min (v(A), v(B)), \\
& v(A \wedge B)=\max (v(A), \quad v(B)) .
\end{aligned}
$$

Throughout this paper, let $p_{1}, \ldots, p_{n}$ be distinct letters and $A$ be such a formula that no letters other than $p_{1}, \ldots, p_{n}$ occur in $A$. Given an $n$-tuple $v\left(p_{1}\right), \ldots, v\left(p_{n}\right)$ of values of $p_{1}, \ldots, p_{n}$, denote, as convention, the letters assigned the value 0 by $r_{1}, \ldots, r_{u}$, the rest by $s_{1}, \ldots, s_{v}$. Then, the following lemma holds for LPS and LOS.

Lemma. For the given n-tuple $v\left(p_{1}\right), \ldots, v\left(p_{n}\right)$ of values of the letters $p_{1}$, $\ldots, p_{n}$

$$
r_{1}, \ldots, r_{u}, \quad s_{1} \rightarrow s_{2}, s_{2} \rightarrow s_{3}, \ldots, s_{v} \rightarrow s_{1} \vdash A^{2)}
$$

or

$$
r_{1}, \ldots, r_{u}, \quad s_{1} \rightarrow s_{1}, s_{2} \rightarrow s_{3}, \ldots, s_{v} \rightarrow s_{1} \vdash A \rightarrow s_{1},
$$

according as $v(A)$ is 0 or 1 .

Let $A$ be a formula of LPS in which no letters other than $p_{1}, \ldots, p_{n}$ occur. Then, we consider the following problem:

How much logical information $\Gamma$ can we deduce $A$ from in LPS?

Of course, we can always deduce $A$ from $p_{1}, \ldots, p_{n}$ in systems of negationless propositional logics.

Now, we restrict the entity of information to such types as $p$ or $p \rightarrow q$. Then, we understand that $\{p\}$ is more (stronger) than $\{q \rightarrow p\}$ and also $\{q, q \rightarrow p\}$ than $\{p\}$ as information (as assumptions). Can we weaken the assumptions $\left\{r_{1}, \ldots, r_{u}, s_{1} \rightarrow s_{2}, \ldots,{ }^{\prime} s_{v} \rightarrow s_{1}\right\}$ to $\left\{r_{n_{1}}, \ldots, r_{n_{i}}, r_{m_{1}} \rightarrow r_{m_{2}}, \ldots, r_{m_{j}} \rightarrow r_{m_{1}}\right.$, $\left.s_{1} \rightarrow s_{2}, \ldots, s_{v} \rightarrow s_{1}\right\}$, where $\left\{r_{n_{1}}, \ldots, r_{n_{i}}, r_{m_{1}}, \ldots, r_{m_{j}}\right\}=\left\{r_{1}, \ldots, r_{u}\right\}, i \geqq 0$ and $i+j=u$, in the lemma? It is impossible, in general. For, take $p$ as $A$ and 0 as the value of $p$, then we would have $p \rightarrow p \vdash p$, which contradicts

2) Throughout this paper, we use ' $\Gamma \vdash A$ ' to express 'A is deducible from $\Gamma$ ' in a system of logic which is arbitrary or clear from context, or in a system of logic specially noticed. 
to the consistency of LPS. As a nontrivial counter example, one may take the sentence $r_{1} \wedge r_{2} \wedge\left(s_{1} \rightarrow s_{2}\right) \wedge\left(s_{2} \rightarrow s_{1}\right)$. In this respect, $\left\{r_{1}, \ldots, r_{u}, s_{1} \rightarrow s_{2}, \ldots\right.$, $\left.s_{v} \rightarrow s_{1}\right\}$ in the lemma is the least information which deduces $A$ in general. That is; our lemma gives not only a sufficient condition but also a necessary condition to solve 'how much?' of the above problem.

This suggests that one may gain a normal form of the propositions in the positive logic. Indeed, we can obtain a normal form theorem in LQS, by making use of this fact and the fact that LQS is complete in the following sense: every formula $A$ in LQS is provable in it if $A$ takes identically the value 0 .

As another corollary to the lemma for LPS (or LOS), we obtain a direct proof ${ }^{3)}$ of the completeness of LQS (resp. LOQS), by making use of the following $^{4)}: p \rightarrow A,(p \rightarrow q) \rightarrow A \vdash A$ in LOQS (a fortiori, in LQS). This is another proof of Curry's one who established the completeness theorem for LQS (in his notation, HC) by reformulating the system in Gentzen's style formalism and using the cut-elimination theorem (see Curry [2], p. 224 also p. 182).

The author expresses his thanks to Prof. Katuzi Ono for kind encourgement and advice given in the preparation of this paper.

\section{The proof of the lemma.}

We prove the lemma for LPS by the induction on the number of logical symbols occurring in $A$ (i.e. on the length of $A$ ). It will be shown in such a manner that the proof as to the case for LOS is automatically contained as a part. However, the latter can be carried out more easily, since the implication $\rightarrow$ is the only logical symbol in LOS. For brevity, we may simply write $\Gamma$ the assumptions $\left\{r_{1}, \ldots, r_{u}, s_{1} \rightarrow s_{2}, \ldots, s_{v} \rightarrow s_{1}\right\}$ below.

BAsIs. There are no logical symbols in $A$, i.e. $A$ is $p_{i}$. Then by the definition of $\Gamma$, we see trivially

$$
\Gamma \vdash p_{i} \quad \text { or } \quad \Gamma \vdash p_{i} \rightarrow s_{1},
$$

according as $v\left(p_{i}\right)$ is 0 or 1 .

InDUCtion Step. There are three cases according to $\rightarrow, \vee$ or $\wedge$ be the outermost logical symol of $A$.

3) After giving the proof, the author found, in Church [1], an exercise which asks to show the completeness of LOQS (in his notation, $P_{B}^{I}$ ) with hint. He uses a selected letter different from $p_{1}, \ldots, p_{n}$ and establishes an analogue of our lemma in LOQS, that is, by making use of Peirce's law. In this respect, our proof seems more cleancut.

4) See Ono [6], Foc tenote 15, p. 341, and also cf. Church [1], 12.8, p. 86. 
Case 1. Let $A$ be $B \rightarrow C$.

Subcase 1.1: $\quad v(B \rightarrow C)=0$. In this case, $v(C)=0$ or $v(B)=1$. When $v(C)=0$, we have $\Gamma \vdash C$ as the hypothesis of the induction. Then we easily see $\Gamma \vdash B \rightarrow C$. When $v(B)=1$, we have

$$
\Gamma \vdash B \rightarrow s_{1}
$$

as the hypothesis of the induction. Now, let us assume $B$. Then we have $s_{1}$. Hence, we have successively $s_{2}, \ldots, s_{v}$, since $s_{1} \rightarrow s_{2}, \ldots, s_{v-1} \rightarrow s_{v}$ are in $\Gamma$. So, we have $\Gamma, B \vdash p_{1}, \ldots, p_{n}$. As is easily seen, $p_{1}, \ldots, p_{n} \vdash C$ holds in the positive logics. Thus, it follows that $\Gamma, B \vdash C$ holds. The latter implies $\Gamma \vdash B$ $\rightarrow C$ by the deduction theorem.

Subcase 1.2: $\quad v(B \rightarrow C)=1$, In this case, $v(B)=0$ and $v(C)=1$. We wish to show $\Gamma \vdash(B \rightarrow C) \rightarrow s_{1}$. Assume $B \rightarrow C$. By the hypothesis of the induction, we have

$$
\Gamma \vdash B \quad \text { and } \quad \Gamma \vdash C \rightarrow s_{1} \text {. }
$$

Using these succesively, we see $\Gamma, B \rightarrow C \vdash s_{1}$. Therefore, it holds that

$$
\Gamma \vdash(B \rightarrow C) \rightarrow s_{1}
$$

Case 2. Let $A$ be $B \vee C$.

Subcase 2.1: $v(B \vee C)=0$. In this case, $v(B)=0$ or $v(C)=0$. By the hypothesis of the induction, $\Gamma \vdash B$ or $\Gamma \vdash C$, respectively. In each case, holds $\Gamma \vdash B \vee C$.

Subcase 2.2: $\quad v(B \vee C)=1$. In this case, $v(B)=1$ and $v(C)=1$. Hence, we have simultaneously $\Gamma \vdash B \rightarrow s_{1}$ and $\Gamma \vdash C \rightarrow s_{1}$ as the hypothesis of the induction. Then it holds that

$$
\Gamma \vdash B \vee C \rightarrow s_{1}
$$

since $B \vee C \rightarrow s_{1}$ is deducible from the formulae $B \rightarrow s_{1}, C \rightarrow s_{1}$.

Case 3. Let $A$ be $B \wedge C$.

Subcase 3.1: $v(B \wedge C)=0$. In this case, $v(B)=0$ and $v(C)=0$. As the hypothesis of the induction, we have $\Gamma \vdash B$ and $\Gamma \vdash C$ simultaneously.- Then, it follows immediately that $\Gamma \vdash B \wedge C$.

Subcase 3.2: $v(B \wedge C)=1$. In this case, holds at least one of $v(B)=1, v(C)=1$. 
Then by the hypothesis of the induction, we see

$$
\Gamma \vdash B \rightarrow s_{1} \quad \text { or } \quad \Gamma \vdash C \rightarrow s_{1} .
$$

On the other hand, $B \wedge C \vdash B$ and $B \wedge C \vdash C$. Therefore, we have $\Gamma, B \wedge C$ $\vdash s_{1}$ in any case, and hence $\Gamma \vdash B \wedge C \rightarrow s_{1}$.

Thus, the proof of the lemma is established.

\section{A normal form of the negationless propositions.}

We give a principal normal form of the formulae $A$ in LQS. For the purpose, let the letters occurring in $A$ be exactly $p_{1}, \ldots, p_{n}$.

In the first place, by the lemma, we see

*)

$$
\underset{v(A)=0}{\bigvee_{1}}\left(r_{1} \wedge \ldots \wedge r_{u} \wedge\left(s_{1} \rightarrow s_{2}\right) \wedge \ldots \wedge\left(s_{v} \rightarrow s_{1}\right)\right) \rightarrow A
$$

is provable in LPS.

where $\underset{v(A)=0}{V}$ means the disjunction of all members $r_{1} \wedge \ldots \wedge r_{u} \wedge\left(s_{1} \rightarrow s_{2}\right) \wedge$ $\ldots \wedge\left(s_{v} \rightarrow s_{1}\right)$ depending on the $n$-tuples of values of $p_{1}, \ldots, p_{n}$ for which $A$ takes the value 0 . In fact, let $A$ take the value 0 for a given $n$-tuple $v\left(p_{1}\right), \ldots, v\left(p_{n}\right)$ of values of the letters $p_{1}, \ldots, p_{n}$. Then, by the lemma, $r_{1}, \ldots, r_{u}, s_{1} \rightarrow s_{2}, \ldots, s_{v} \rightarrow s_{1} \vdash A$, i.e. $r_{1} \wedge \ldots \wedge r_{u} \wedge\left(s_{1} \rightarrow s_{2}\right) \wedge \ldots \wedge$ $\left(s_{v} \rightarrow s_{1}\right) \vdash A$ holds. It follows that $\left(r_{1} \wedge \ldots \wedge r_{u} \wedge\left(s_{1} \rightarrow s_{2}\right) \wedge \ldots \wedge\left(s_{v} \rightarrow s_{1}\right)\right)$ $\rightarrow A$ is provable in LPS. Hence, we have $\left.{ }^{*}\right)$.

Coversely, $A \rightarrow \underset{v(A)=0}{\bigvee}\left(r_{1} \wedge \ldots \wedge r_{u} \wedge\left(s_{1} \rightarrow s_{2}\right) \wedge \ldots \wedge\left(s_{v} \rightarrow s_{1}\right)\right)$ takes identically the value 0 . For, if $A$ takes the value 0 for any given $n$-tuple $v\left(p_{1}\right), \ldots$, $v\left(p_{n}\right)$, then the corresponding disjundctive member $r_{1} \wedge \ldots \wedge r_{u} \wedge\left(s_{1} \rightarrow s_{2}\right) \wedge$ $\ldots \wedge\left(s_{v} \rightarrow s_{1}\right)$ also takes the value 0 by the convention for $\left\{r_{1}, \ldots, r_{u}\right\}$ and $\left\{s_{1}, \ldots, s_{v}\right\}$. Hence, by making use of the completeness of LQS, this is provable in LQS.

Now, we say that $A$ is equivalent to $B$ in LQS, if both the formulae $A \rightarrow B$, $B \rightarrow A$ are theorems of LQS. We have obtained the following:

Theorem. Let $A$ be any formula in LQS and $p_{1}, \ldots, p_{n}$ be the letters occurring in $A$. Then $A$ is equivalent to the formula

$$
\underset{v(A)=0}{\bigvee}\left(r_{1} \wedge \ldots \wedge r_{n} \wedge\left(s_{1} \rightarrow s_{2}\right) \wedge \ldots \wedge\left(s_{v} \rightarrow s_{1}\right)\right)
$$

in LQS. 
Thus, any proposition $A$ in the positive logics is rewritten in the form **) (which can be uniquely determined to within the order of its disjunctive members) in LQS, by using only the letters occurring in $A$. Therefore, the latter is competent for the principal normal form of the negationless propositions.

By the above result, we can say LQS is also notationally complete in the folowing sense: each of the $2^{2^{n}-1}$ possible positive propositional functions of $n$-variables $p_{1}, \ldots, p_{n}$ can be represented by a formula in these letters ${ }^{5}$.

Notice: Of course, we cannot express the propositions by so-called disjunctive (or conjunctive, either) principal normal form in LQS. On the other hand, our normal form theorem is not true for the classical sentential logic LKS. In this sense, the theorem gives a characterization of LQS.

\section{REFERENCES}

[1] A. Church, Introduction to mathematical logic, I, Princeton, 1956.

[2] H.B. Curry, Foundations of mathematical logic, New York, 1963.

[3] P. Lorenzen, Einführung in die operative Logik und Mathematik, Berlin-Göttingen-Heidelberg, 1955.

[4] L. Kalmár, Über die Axiomatisierbarkeit des Aussagenkalküls, Acta Univ. Szeged, 7 (1934-5), 222-243.

[5] S.C. Kleene, Introduction to metamathematics, Amsterdam-Groningen and New YorkTronto, 1952.

[6] K. Ono, On universal character of the primitive logic, Nagoya Math. J., 27 (1966), 331 353.

[7] — A formalism for the classical sentence-logic, Nagoya Math. J. (in printing).

Nagoya University

5) See e.g. Kleene [5], p. 135. 